\title{
Influencia del aumento de la densidad y nivel de adhesivo en las propiedades mecánicas de tableros de partículas de Chusquea culeou. aglomerados con ureaformaldehído
}

\author{
Influence of the increase density and resin level on mechanical properties \\ of Chusquea culeou particleboard agglomerated with ureaformaldehyde \\ C.D.O.: $832.2: 812.7$
}

CARLOS RIJO

Avda. Fernando de Navarrete 119, Santo Domingo, República Dominicana

\section{SUMMARY}

The effect of increasing solid resin, and the density of manufactured Chusquea culeou Desv. particleboard, agglomerated with ureaformaldehyde was studied.

The one-layer boards were $11 \mathrm{~mm}$ thick, and were manufactured using shaves like those used in coke layers. The Densities studied were $450,550,650$ and $750 \mathrm{Kg} / \mathrm{m}^{3}$ and the resin levels $6,7,8,9$ and $10 \%$ based on oven-dry shave weigth.

Mechanical properties were tested according to DIN standard, and were analized by linear regression. A high correlation between studied mechanical properties and density was found.

The adhesive effect was significant for the bending strength only over a $600 \mathrm{Kg} / \mathrm{m}^{3}$ density and with a resin level over $8 \%$. Concerning internal bond, the increase resin level is advantageous in all studied densities.

\section{RESUMEN}

Se estudia el efecto al incrementar el nivel de resina sólida como de aumentar la densidad en tableros de partículas elaboradas con virutas de Chusquea culeou Desv., aglomerados con ureaformaldehído.

Los tableros se elaboraron de una sola capa, con virutas del tipo capa media, con un espesor de 11 mm. Las densidades estudiadas correspondieron a $450 ; 550 ; 650$ y $750 \mathrm{~kg} / \mathrm{m}^{3} ;$ los niveles de adhesivos estudiados fueron 6 , 7,8 , 9 y $10 \%$, basado en el peso anhidro de las virutas.

Los ensayos de las propiedades mecánicas se realizaron de acuerdo con las normas DIN y se analizaron mediante regresión lineal; se encontró un alto grado de correlación entre las propiedades mecánicas estudiadas y la densidad.

El efecto del adhesivo sobre la flexión fue significativo en los tableros de densidad superior a $600 \mathrm{~kg} / \mathrm{m}^{3} \mathrm{y} \mathrm{con}$ niveles de adhesivo mayores a $8 \%$. En la resistencia a la tracción, el aumento del nivel de adhesivo es positivo en todos los niveles de densidad estudiados.

De acuerdo con los resultados Chusquea culeou Desv. puede ser incluida en los tableros de partículas con densidades superiores a $600 \mathrm{~kg} / \mathrm{m}^{3}$ y con niveles de adhesivo superiores al $8 \%$.

\section{INTRODUCCION}

La especie bambuseae coligüe (Chusquea culeou Desv.) se desarrolla naturalmente en Chile desde Molina $\left(35^{\circ} \mathrm{S}\right)$ al Lago General Carrera $\left(46^{\circ} 30^{\prime} \mathrm{S}\right)$, entre los 500 a 1.000 m.s.n.m., de preferencia en la Cordillera de los Andes, constituyendo principalmente el sotobosque (Haverbeck, 1983). En estas condiciones la especie tiene una producción primaria neta del orden de 10-11 ton/há/año (Veblen, Schlegel y Escobar, 1979).
La alta producción primaria de coligüe permite aseverar que su utilización industrial podría ser rentable.

Además de su gran producción primaria, la vasta distribución del coligüe posibilita el establecimiento de una industria de tableros de partículas, la cual podría fundamentarse en la utilización de los culmos cosechados en el sotobosque, como desecho del manejo forestal e incluso aplicar las medidas silviculturales que correspondan para posibilitar la cosecha de 
los culmos basándose en el concepto de rendimiento sostenido.

Pensando en la posibilidad de utilizar coligüe en la producción de tableros se desarrolló este trabajo. En él se pretende determinar su calidad como materia prima para la fabricación de estos aglomerados.

Además, el estudio tiene como objetivo determinar la influencia de un aumento de la densidad en tableros elaborados con esta especie, así como variaciones de los niveles de adhesivo, sobre las propiedades mecánicas, flexión y tracción.

\section{METODO EXPERIMENTAL}

Para la realización del estudio se utilizaron culmos de edad superior a un año, con lo cual se garantizó que hubiese finalizado su proceso de lignificación (Itoh y Shemajii, $1981)$.

Los culmos de coligüe fueron transformados en virutas en un viruteador de cuchillos sobre cilindro de tamaño industrial.

Una vez elaboradas las virutas, éstas fueron secadas hasta un contenido de humedad adecuado al proceso; este contenido de humedad fluctuó entre 4 y $6 \%$. Posterior al secado las virutas fueron tamizadas, eliminándose el material grueso y el polvo.

Las virutas fueron tamizadas mediante vibración, lo que permitió determinar las características de éstas, así como la participación porcentual de cada tamaño. Los tamaños de viruta, su longitud, su espesor promedio y coeficiente de esbeltez se muestran en el Cuadro 1.

Este tipo de virutas, de acuerdo a sus características, corresponde al usado normalmente en la industria para capa media.

Las virutas fueron encoladas con niveles de ureaformaldehído de $6,7,8,9$ y $10 \%$, calculado en base al peso seco de las virutas. La ureaformaldehído fue catalizada con cloruro de amonio $\left(\mathrm{NH}_{4} \mathrm{C} 1\right)$ a razón de $1,5 \%$ del peso seco de la resina.

Los tableros fueron confeccionados con un espesor de $11 \mathrm{~mm}$ de una sola capa y con densidades calculadas de 450, 550, 650 y $750 \mathrm{~kg} / \mathrm{m}^{3}$.

El prensado de los tableros se realizó siguiendo el esquema mostrado en la Fig. 1. La temperatura se mantuvo constante en $160^{\circ} \mathrm{C}$.

La obtención de las probetas para los ensayos de tracción (DIN 52365) y flexión (DIN 52362) se realizó de acuerdo con una matriz que permite una individualización y ubicación similar de cada probeta dentro del tablero.

Previo a ser ensayadas, las probetas fueron climatizadas de acuerdo a las condiciones establecidas por DIN 50014.

En las probetas para el ensayo de flexión se determinó la densidad según DIN 52361. Para las probetas de tracción la densidad se determinó en la probeta completa, previo a ser ensayada, ya que, una vez realizado el

\section{CUADRO 1}

Características de las astillas de Chusquea culeou.

Characteristics of Chusquea culeou shaves.

\begin{tabular}{|c|c|c|c|c|c|}
\hline \multicolumn{2}{|c|}{$\begin{array}{l}\text { Tamaño } \\
(\mathrm{mm})\end{array}$} & \multirow{2}{*}{$\begin{array}{c}\begin{array}{c}\text { Participación } \\
(\%)\end{array} \\
14,9\end{array}$} & \multirow{2}{*}{$\begin{array}{c}\begin{array}{c}\text { Longitud } \\
(\mathrm{mm})\end{array} \\
24,2\end{array}$} & \multirow{2}{*}{$\begin{array}{c}\begin{array}{c}\text { Espesor } \\
(\mathrm{mm})\end{array} \\
0,47\end{array}$} & \multirow{2}{*}{$\begin{array}{c}\text { Coeficiente esbeltez } \\
51,50\end{array}$} \\
\hline$>$ & 4,0 & & & & \\
\hline $4,0-$ & 2,8 & 11,7 & 23,9 & 0,59 & 40,51 \\
\hline $2,8-$ & 2,0 & 12,8 & 18,8 & 0,55 & 34,18 \\
\hline $2,0-$ & 1,0 & 31,5 & 20,6 & 0,44 & 46,82 \\
\hline $1,0-$ & 0,5 & 23,4 & 17,1 & 0,30 & 57,00 \\
\hline$<$ & 0,5 & 5,7 & 10,5 & 0,17 & 61,76 \\
\hline \multicolumn{3}{|c|}{ Promedio ponderado } & $19.9^{1}$ & $0,43^{1}$ & $48,17^{1}$ \\
\hline
\end{tabular}

1 Los promedios corresponden a la sumatoria de los valores ponderados por su participación porcentual.

Averages correspond to total value weigth by its percentage of participation. 


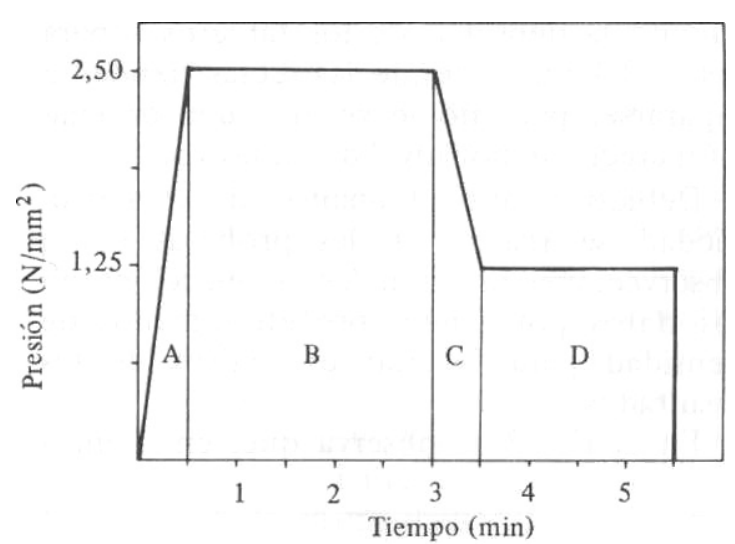

Fig. 1: Diagrama del ciclo de prensado. La temperatura se mantuvo constante a $160^{\circ} \mathrm{C}$.

A: Tiempo de cierre; B: Tiempo presión máxima; C: Tiempo para descender a presión media; D: Tiempo a presión media.

Scheme of pressing cycle. Pressing temperature kept constant at $160^{\circ} \mathrm{C}$.

A: Closure time; B: Time at maximum pressure; C: Time to descend to mean pressure; D: Time at mean pressure. ensayo, ésta se destruye. Estas determinaciones de densidad permitieron correlacionar las propiedades mecánicas con la densidad.

\section{RESULTADOS}

Resistencia a la flexión. Un total de 540 observaciones de densidad y flexión permitieron realizar un análisis de correlación, mediante regresión lineal. Los resultados de este análisis se muestran en la Fig. 2.

Al analizar la Fig. 2 se observa una alta correlación entre la resistencia a la flexión respecto de la densidad. De acuerdo a estos resultados, la densidad puede tomarse como un indicador del valor aproximado que pueda obtenerse en el ensayo de flexión. Esta tendencia ya ha sido demostrada anteriormente por otros investigadores (Vital et al., 1974; Poblete, 1979 y 1985; Urzúa y Poblete, 1980).

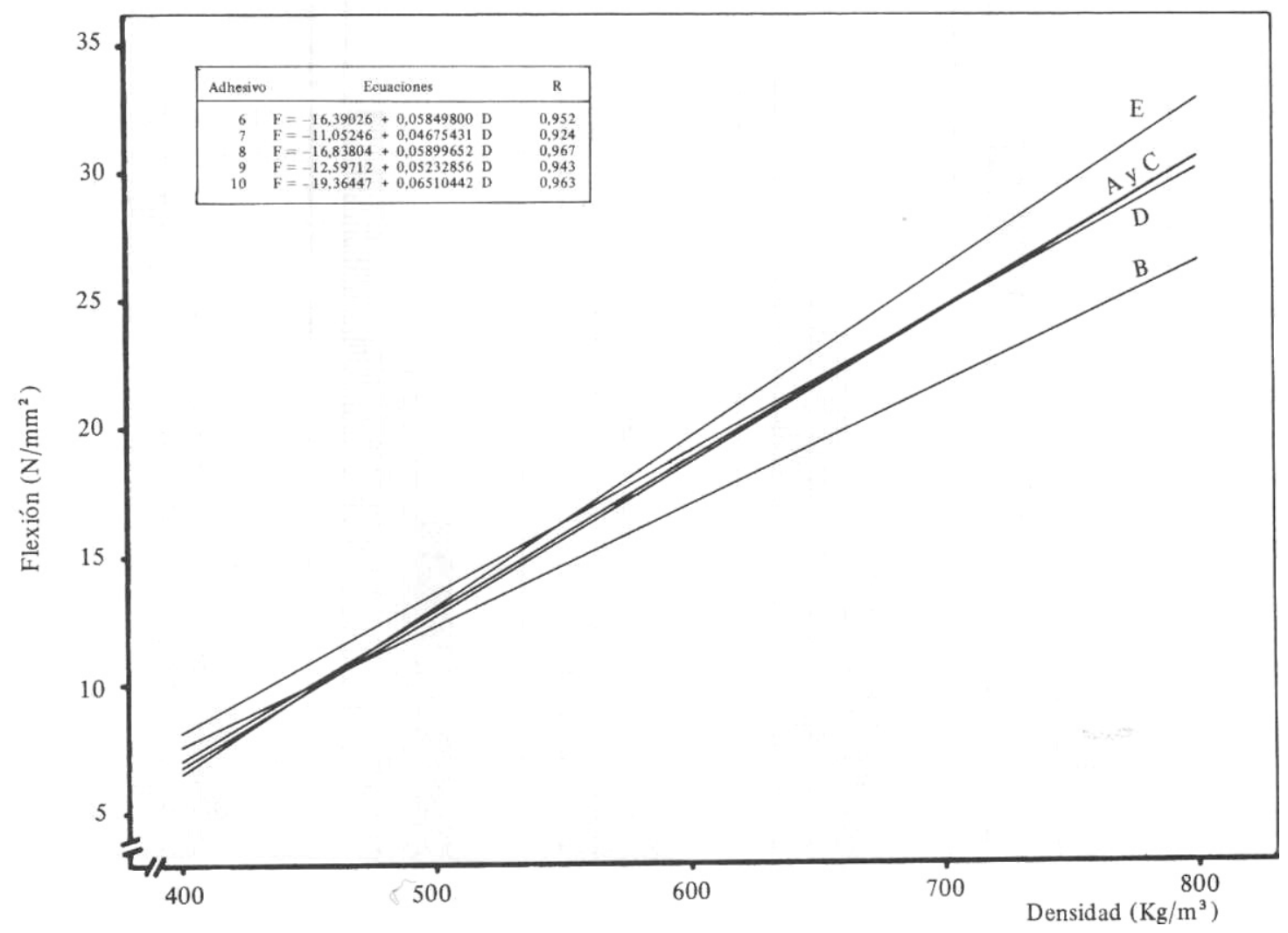

Fig. 2: Variación de la resistencia a la flexión según densidad y nivel de adhesivo.

Nivel de adhesivo: $\mathrm{A}=6 \% ; \mathrm{B}=7 \% ; \mathrm{C}=8 \% ; \mathrm{D}=9 \% ; \mathrm{E}=10 \%$.

Variation of the bending strength according to density and adhesive level. Adhesive level: $\mathrm{A}=6 \% ; \mathrm{B}=7 \% ; \mathrm{C}=8 \% ; \mathrm{D}=9 \% ; \mathrm{E}=10 \%$. 
No se observa un aumento del coeficiente de determinación $\left(\mathrm{R}^{2}\right)$, que pueda ser atribuido al aumento del contenido de ureaformaldehído en el panel; sin embargo, se destaca que el nivel de adhesivo $8 \%$ registra los mejores parámetros para la regresión.

Se aprecia que las rectas no presentan gran diferencia en su punto de origen, lo que indica que el efecto del contenido de adhesivo es bajo. Este defecto se manifiesta cuando la densidad de los tableros supera los $600 \mathrm{~kg} / \mathrm{m}^{3}$, donde las rectas tienden a separarse, pero no se verifica una ordenación creciente por nivel de adhesivo.

Debido a que el análisis de esta propiedad se realizó en las probetas como observaciones individuales, se seleccionaron los datos por rangos predeterminados de densidad para facilitar el análisis de los resultados.

En la Fig. 3 se observa que, en algunos

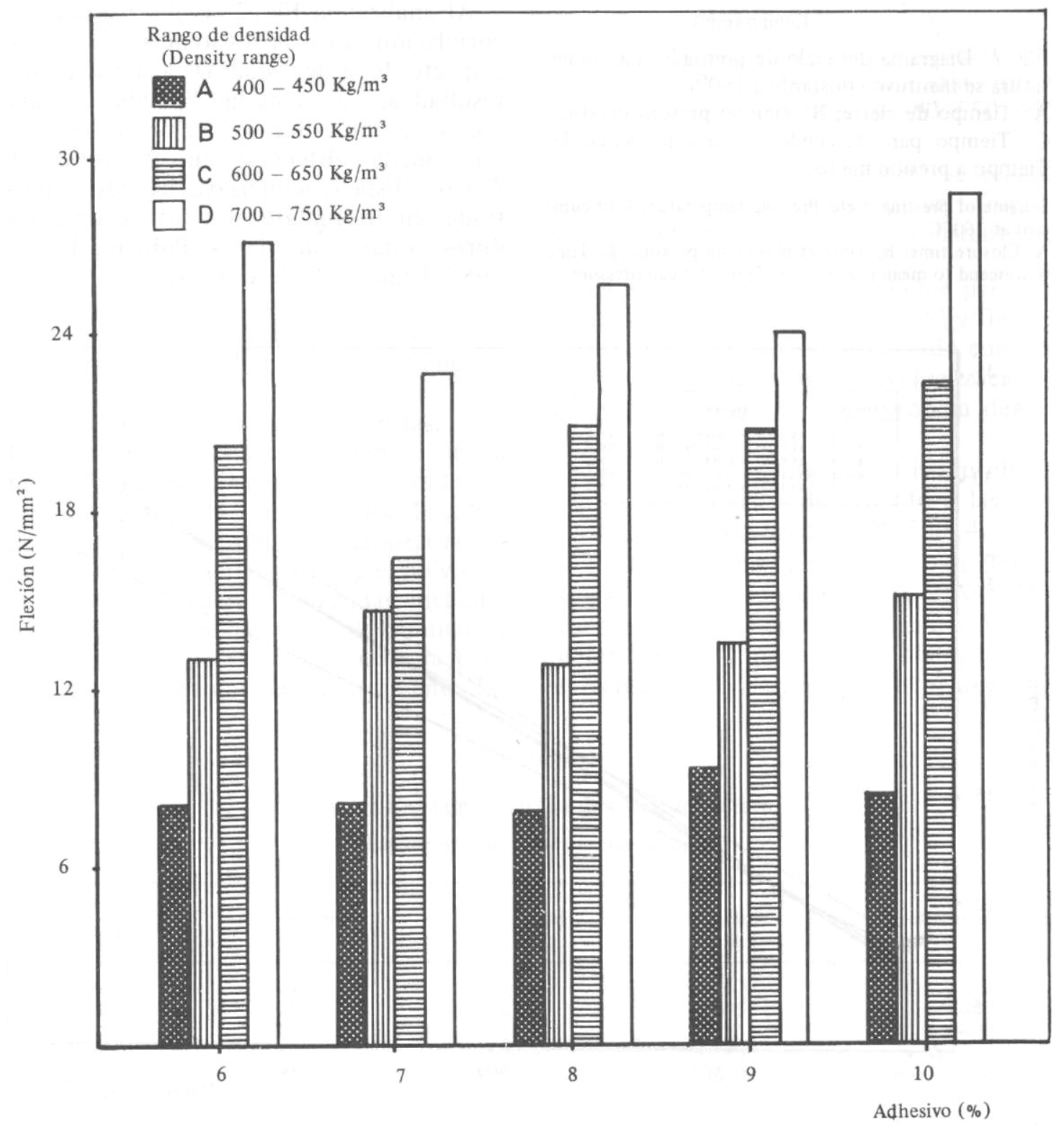

Fig. 3: Valores promedios de resistencia a la flexión, para cada nivel de adhesivo y rango de densidad.

Average values of bending strength, for each adhesive level and density range. 
casos, un aumento del contenido de resina en el tablero provoca una disminución de la flexión. Este hecho ha sido destacado por Shuler (1974), citado por Kelly (1977), quien varió el nivel de ureaformaldehído de 2 a $12 \%$, concluyendo que cuando el nivel de adhesivo es superior a $5 \%$ no se logran aumentos considerables en esta propiedad mecánica.

Es importante destacar la razón de compresión. Según Vital et al. (1974), cuando este cuociente es superior a 1,2 se logran mejores propiedades físicas y mecánicas; para coligüe, esta razón de compresión se logra a partir de $\operatorname{los} 700 \mathrm{~kg} / \mathrm{m}^{3}$ de densidad en el tablero, ya que la densidad de la especie es de $584 \mathrm{~kg} / \mathrm{m}^{3}$ (Rijo et al., 1987). No obstante lo anterior, en las densidades comprendidas entre 600-650 $\mathrm{kg} / \mathrm{m}^{3}$, donde la razón de compresión es de 1,03-1,11, puede observarse una tendencia de la flexión a aumentar a partir del nivel de adhesivo 8\%. Esto permite aseverar que para tableros de coligüe se logran las propiedades mecánicas mínimas entre las razones de compresión señaladas.

La norma DIN 68761, tomada como patrón de comparación, exige un valor mínimo para esta propiedad de $18 \mathrm{~N} / \mathrm{mm}^{2}$; de la figura 3 puede deducirse que este requisito se cumple con densidades superiores a $600 \mathrm{~kg} / \mathrm{m}^{3}$ y un nivel de adhesivo $8 \%$. Cuando la densidad es superior a $700 \mathrm{~kg} / \mathrm{m}^{3}$ (razón de compresión 1,2) todos los niveles de adhesivos estudiados permiten cumplir con los requisitos de la norma.

Resistencia a la tracción. Para esta propiedad se realizó un total de 689 observaciones, y se efectuó un análisis de regresión lineal, determinándose que existe un alto grado de correlación entre la resistencia a la tracción y la densidad. Estos resultados son coincidentes con los de otros autores (Vital et al., 1974; Poblete, 1979 y 1985; Urzúa y Poblete, 1980).

El alto grado de correlación existente entre la tracción y la densidad se ve mejorado en términos generales, al incrementar el contenido de resina sólida en el tablero. Este efecto se determinó al verificarse un aumento del coeficiente de determinación $\left(\mathrm{R}^{2}\right)$ entre los niveles 8 a $10 \%$, siendo mayor con 9\% de adhesivo, donde los demás parámetros de la regresión son los mejores para la ecuación estimadora de tracción.

Las rectas identificadas por las ecuaciones de regresión se muestran en la Fig. 4.

En la Fig. 4 se visualiza igualmente el efecto de la densidad del tablero sobre la resistencia a la tracción. Las rectas presentan diferencias desde su origen, las que se acentúan con el aumento de la densidad; esto demuestra un efecto combinado del nivel de adhesivo y la densidad. El efecto más significativo está representado por la densidad, registrándose un aumento porcentual que varía entre 19 y $56 \%$ al pasar de un nivel de densidad a otro.

En la Fig. 5 se aprecia un aumento de la resistencia a la tracción al aumentar el contenido de adhesivo; esto es válido para todos los rangos de densidades estudiados, observándose más claramente a partir de los $500 \mathrm{~kg} / \mathrm{m}^{3}$.

La tendencia registrada por esta propiedad mecánica, respecto al nivel de adhesivo, se explica por ser ésta una medida de la calidad de unión interna de las virutas. Los resultados aquí obtenidos coinciden con otros estudios (Larmore, 1959; Gatchel et al. 1966; Lehmann, 1970).

La norma DIN 68761 exige un mínimo de $0,4 \mathrm{~N} / \mathrm{mm}^{2}$ para esta propiedad en tableros con espesores comprendidos en los límites en que se confeccionaron los de este estudio. Estas exigencias se logran a partir de $600 \mathrm{~kg} / \mathrm{m}^{3}$, con todos los niveles de adhesivo estudiados; cuando el nivel de adhesivo es de 7\%, o superior, las densidades de $500 \mathrm{~kg} / \mathrm{m}^{3}$ permiten cumplir con dichas exigencias.

\section{CONCLUSIONES}

Los resultados obtenidos permiten concluir que:

- Chusquea culeou Desv. es técnicamente factible de ser incluida en tableros de partículas.

- En tableros de partículas de Chusquea culeou Desv., encolados con ureaformaldehído, existe un alto grado de correlación entre las propiedades mecánicas, resistencia a la flexión y resistencia a la 


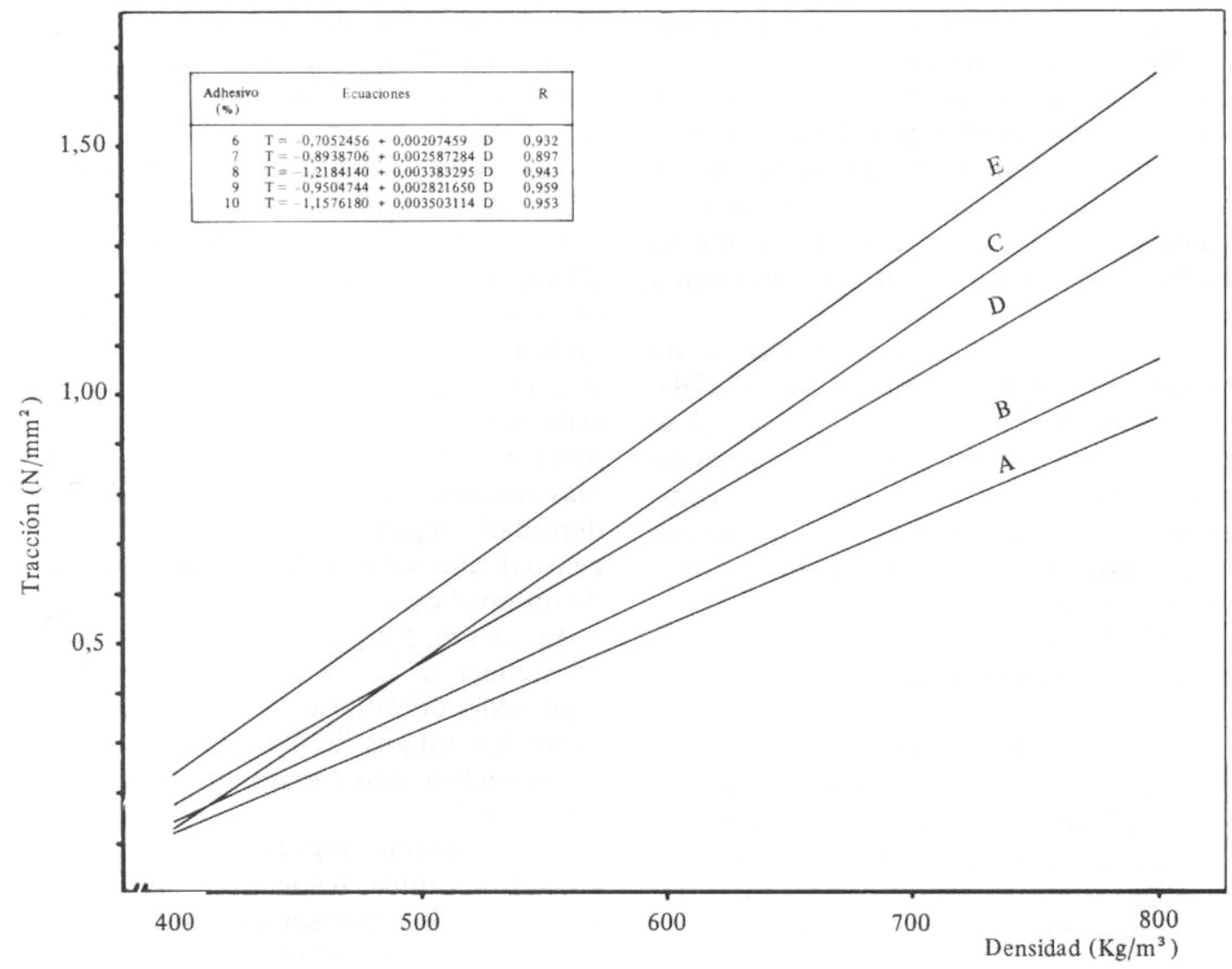

Fig. 4: Variación de la resistencia a la tracción según densidad y nivel de adhesivo.

Variation of the internal bond according to density and adhesive level.

Nivel de adhesivo: $\mathrm{A}=6 \% ; \mathrm{B}=7 \% ; \mathrm{C}=8 \% ; \mathrm{D}=9 \% ; \mathrm{E}=10 \%$.

Adhesive level: $\mathrm{A}=6 \% ; \mathrm{B}=7 \% ; \mathrm{C}=8 \% ; \mathrm{D}=9 \% ; \mathrm{E}=10 \%$.

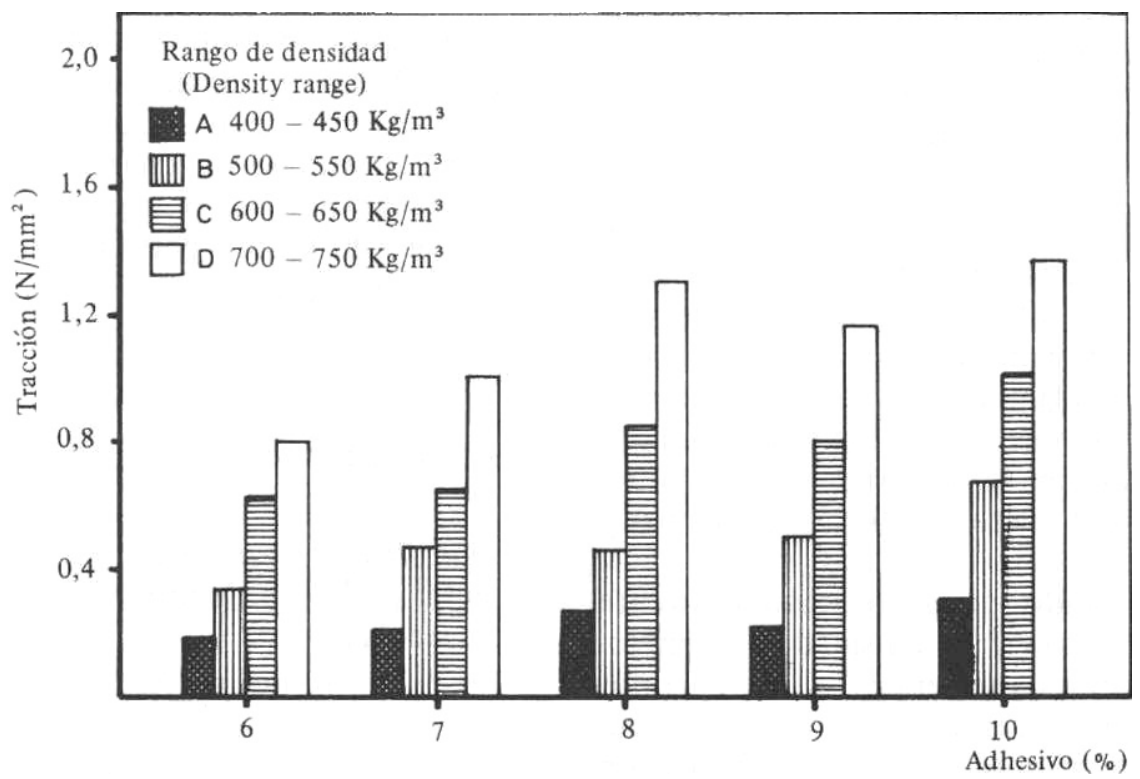

Fig. 5: Valores promedios de la resistencia a la tracción por rango de densidad y niveles de adhesivo. Average values of internal bond by density range, and adhesive levels. 
tracción, con respecto a la densidad del panel.

- En la tracción el efecto de la densidad puede verificarse en un mejoramiento de esta propiedad que varía entre 19 a $56 \%$ al pasar de un nivel de densidad a otro, con el mismo nivel de adhesivo.

- Un aumento del nivel de adhesivo favorece la resistencia a la flexión sólo en los niveles de densidad superior a 600 $\mathrm{kg} / \mathrm{m}^{3}$. Este efecto fue más significativo al pasar de los niveles 8 a 9 y $10 \%$ en el rango de densidad señalado.

- En el caso de la tracción, el efecto positivo de aumentar el nivel de adhesivo sobre esta propiedad se manifiesta en todas las densidades estudiadas.

\section{LITERATURA CITADA}

GATCHEL, C; HEEBINK, B. y HEFTY, F. 1966. "Influence of components variables on properties of particleboard for exterior use". For. Prod. J. 16(4): 46-59.

HAVERBECK, M. 1983. Estudio de crecimiento, variación morfológica y reacción al corte de coligüe (Chusquea culeou Desv.) en un bosque de Coigüe-Tepa-Mañio, en el predio San Pablo de Tregua, Panguipulli. Tesis de grado, Fac. Cs. Forestales, Universidad Austral de Chile. Valdivia, $124 \mathrm{pp}$.

ITOH, T. y SHEMAJII, K. 1981. "Lignification of bamboo culm (Phyllostachys pubescens) during its growth and maturation". Proceedings of the
Congress Group 5.3 A. Production and utilization of bamboo and related species. XVII IUFRO World Congress, Kyoto, Japan, 6-17 septiembre/81.

KELLY, M. 1977. "Critical literature review of relationships between processing parameter and physical properties of particleboard". General Technical Report FPL -10, Madison, 64 pp.

LARMORE, F. 1959. "Influence of specific gravity and resin content on properties of particleboard". For. Prod. J. 9(4): 131-134.

LEHMANN, W. 1970. "Resin efficiency in particleboard as influenced by density, atomization, and resin content". Prod. For. J. 20(1): 48-54.

POBLETE, H. 1979. "Inclusion de aserrín en tableros de partículas". Bosque 3(1): 39-46.

1985. "Influencia del tamaño de partículas sobre las propiedades mecánica, flexión y tracción en tableros de partículas aglomeradas con ureformaldehído". Simposio Pinus radiata. Investigación en Chile. Universidad Austral de Chile. Fac. Cs. Forestales. Tomo II pp. 241-256.

RIJO, C; POBLETE, H.; DIAZ-VAZ, J.E.; TORRES, M.; FERNANDEZ, A. 1987. "Estudio de algunas características anatómicas, físicas y químicas de Chusquea culeou (Coligüe)". Bosque 8(1): 59-61.

URZUA, D. y POBLETE, H. 1980. Utilización de los terrenos de Nadi. Informe $N^{\circ}$ 2. Factibilidad técnica de la producción de tableros de partículas utilizando especies que crecen en los terrenos de Nadi. Convenio SERPLAC X Región-UACH. Informe $\mathrm{N}^{\circ} 29.140 \mathrm{pp}$.

VEBLEN, T.; SCHLEGEL, y ESCOBAR, B. 1979. "Biomasa y producción primaria de Chusquea culeou Desv. y Chusquea tenniflora Phil, en el sur de Chile". Bosque 3(1): 47-56.

VITAL, B.; LEHMANN, W. y BOONE, R. 1974. "How species and board densities affect properties of exotic hardwood particleboard". For. Prod. J. 24(12): $37-45$. 